\title{
Interfacial Instability of Thixotropic Fluids in Triple-Layered Channel Flow
}

\author{
M. Safarifard ${ }^{1}$, M. Pourjafar ${ }^{2}$ and K. Sadeghy ${ }^{1 \dagger}$ \\ ${ }^{1}$ Center of Excellence in Design and Optimization of Energy Systems (CEDOES), School of Mechanical \\ Engineering, College of Engineering, University of Tehran, Tehran, Iran \\ ${ }^{2}$ Department of Mechanical Engineering, University of Tehran, Rezvanshahr, 43841-119, Iran
}

†Corresponding Author Email: sadeghy@ut.ac.ir

(Received December 30, 2019; accepted February 12, 2020)

\begin{abstract}
Interfacial stability of purely-viscous fluids is numerically investigated in channel flow. It is assumed that the main fluid (i.e., the fluid flowing through the center of the channel) is thixotropic and obeys the Moore model as its constitutive equation while the fluids flowing above and below this central (core) layer are assumed to be the same Newtonian fluids with the same thickness. Having found an analytical solution for the base-flow in all three layers, a temporal, normal-mode, linear stability analysis is employed to investigate the vulnerability of the base flow to small perturbations. An eigenvalue problem is obtained this way which is solved numerically using the pseudo-spectral collocation method. The main objective of the work is to explore the role played by the time-constant introduced through the core fluid's thixotropic behavior on the critical Reynolds number. It is found that the thixotropic behavior of the core fluid has a stabilizing effect on the interface. An increase in the viscosity of the upper/lower Newtonian fluids is predicted to have a stabilizing or destabilizing effect on the interface depending on the parameter values of the Moore model (e.g., the ratio of the zero-shear viscosity to infinite-shear viscosity in this fluid model).
\end{abstract}

Keywords: Core-annular flow; Interfacial instability; Thixotropy; Spectral method; Moore model.

\section{NOMENCLATURE}

$\begin{array}{ll}\mathrm{a}, \mathrm{b} & \text { parameters of the Moore model } \\ \mathrm{Cr} & \text { density ratio } \\ \mathrm{C}_{\mu} & \text { cross-viscosity ratio } \\ \mathrm{G} & \text { pressure gradient } \\ \mathrm{H} & \text { channel's half-height } \\ \mathrm{N} & \text { number of Chebyshev terms } \\ \mathrm{p} & \text { pressure } \\ \mathrm{R} & \text { thickness ratio } \\ \mathrm{Re} & \text { Reynolds number } \\ \mathrm{u} & \text { streamwise velocity } \\ \mathrm{v} & \text { cross-stream velocity } \\ \mathrm{x} & \text { axial coordinate }\end{array}$

\section{INTRODUCTION}

Instability of the interface between two or more fluid layers in direct contact with each other comprises one of the most important examples of instability in the field of fluid mechanics. This kind of instability is frequently encountered in

$\begin{array}{ll}\mathrm{y} & \text { vertical coordinate } \\ \Phi & \text { dimensionless rebuild parameter } \\ \alpha & \text { viscosity-difference ratio } \\ \delta & \text { deflection of interface } \\ \kappa & \text { wave number } \\ \lambda & \text { structural parameter } \\ \mu & \text { viscosity } \\ \rho & \text { density } \\ \omega & \text { phase speed } \\ \zeta & \text { thixotropy number }\end{array}$

several industrially-important processes such as coextrusion which is widely used for the production of multilayered plastic sheets and pipes (Schrenk et al. 1978; Anturkar et al. 1990a; Hinch et al. 1992; Chen and Zhang, 1993). It is also encountered in a variety of upstream and downstream operations in oil industry. One can mention, for example, enhanced 
oil recovery (EOR) operations where the huge viscosity stratification at the interface between the displacing fluid (say, water) and the crude oil gives rise to finger instability severely limiting the rate of oil production (Saffman and Taylor, 1958). Another example is the pipeline transport of heavy crude oils where to lower the pressure drop, a low-viscosity fluid such as water or kerosene is injected near the wall to form core-annular flow (Clark and Shapiro, 1949). In practice, however, the interface easily becomes unstable severely affecting effectives of the operation (Joseph et al. 1997; Ghosh et al. 2009).

Another important operation which is severely affected by the interfacial instability is the cementing of oil wells (Taghiloo et al. 2019). In this operation, after drilling a borehole and inserting the steel casing inside the well, cement slurry is pumped into the casing such that it can fill/seal the annular gap between the casing and the rock formation surrounding the casing. During this operation, the cement slurry has to displace the drilling mud collected at the bottom of the well. In practice, however, due to density and viscosity stratification between the two liquids, we might witness finger instability which deteriorates cement quality thereby severely affecting its sealing efficiency (Foroushan et al. 2018).

Due to its technological impact, interfacial instability has been the subject of many studies in the past $(\mathrm{Hu}$ and Joseph, 1989; Preziosi et al. 1990; Chen and Joseph, 1991; Sahu, 2019). These studies have been primarily concerned with Newtonian fluids. And, they have shown that viscosity stratification is the main cause of instability in the above-mentioned applications. As is well-established in the literature (Yih 1967; Yu et al. 1969; Hickox 1971; Hooper et al. 1983) any discontinuity in the viscosity of the two fluids gives rise to a discontinuity in the slope of the velocity profile and this leads to an unstable distribution of vorticity at the interface. In practice, the instability exhibits itself by the interface becoming wavy-shaped. And, as soon as it becomes wavy-shaped surface tension becomes involved (through boundary conditions) provided the two fluids are immiscible. Under these conditions, it has been well established in the literature that surface tension stabilizes all wavenumbers larger than a threshold in viscosity-driven interfacial instability of long interfaces (Bellman and Pennington, 1954).

Although the studies mentioned above have shed some light onto our understanding of the interfacial instability, it must be said that these works have been primarily concerned with Newtonian fluids only. Industrial fluids, however, are often realized not to be Newtonian. For example, waxy crude oils are known to exhibit a variety of non-Newtonian behavior such as shear-thinning, viscoelasticity, and thixotropic behavior (Martinez-Palou et al. 2011; Wardhaugh and Boger, 1991; Kan et al. 2004). The question then arises as to the role played by the rheological properties of the core fluid on the interfacial instability.

In two excellent works, Renardy (1988) and Chen (1991) showed that stratification in the elasticity of the two fluids at the interface can be responsible for the rise of this kind of instability even when there is no density or viscosity stratification involved. In another interesting work, Su and Khomami (1991) investigated the role played by shear-thinning on the instability of core-annular flow and discovered that shear-thinning has a drastic effect on the interfacial stability. There are also several works addressing the effect of a fluid's yield stress on the instability of core-annular flow. One can particularly mention the work by Hormozi et al. (2011) who showed that yield stress can be used as a passive means to stabilize the interface between cement slurry and drilling mud (even if they are viscoelastic) as encountered in the cementing operation of oilwells; see, also, Foroushan et al. (2018). To the best of our knowledge, however, the effect of a fluid's thixotropic behavior on the interfacial instability in core-annular flow has not been addressed in the past. This is surprising realizing the fact that thixotropy is quite common among industrial and physiological fluids (Mewis 1979; Barnes 1997). In fact, thixotropy appears to be a rule rather than exception among fluids such as waxy crude oils (Ahmadpour and Sadeghy, 2014), drilling muds (Livescu, 2012), and cement slurry (Clement, 1979).

In this study, we intend to investigate the effect of a fluid's thixotropic behavior on the stability of its interface with a Moore fluid sandwiched between two Newtonian fluid layers where Newtonian fluids are of the same viscosity and have the same height. The applicability of triple-layered fluids for stabilizing core fluid has been demonstrated in recent years (Sarmadi and Friggard, 2019). For ease of analysis, we assume that the thixotropic fluid flowing through the core of the channel obeys the Moore model (Moore, 1959; Cheng et al. 1965). With the same token, like Renardy (1985), and Anturkar et al. (1990b), we rely on a two-dimensional model as the flow geometry. Our main objective is to investigate the role played by the "characteristic time" of the fluid on the instability of the interface. The prime motivation for conducting this work arises from our interest in the cementing operation of drilled boreholes (Taghiloo et al. 2019) where cement slurry and drilling muds are known to be slightly miscible but not fully mixed (Frigaard and Crawshhaw, 1999).

The work is organized as follows. In the next section we present the mathematical formulation in its unsteady form which can be used to find the base flow under steady conditions. We then proceed with developing the eigenvalue problem governing the unstable modes followed by the numerical method used to solve these differential equations. Numerical results are presented next accompanied by discussing their significance. The work is concluded by presenting a summary of its major findings and their implications in the oil industry and core-annular flow.

\section{MATHEMATiCAL FORMULATiON}

We consider pressure-driven flow of a triple-layered binary fluid system in a plane channel under laminar conditions. This geometry closely models the situation encountered for the production of 


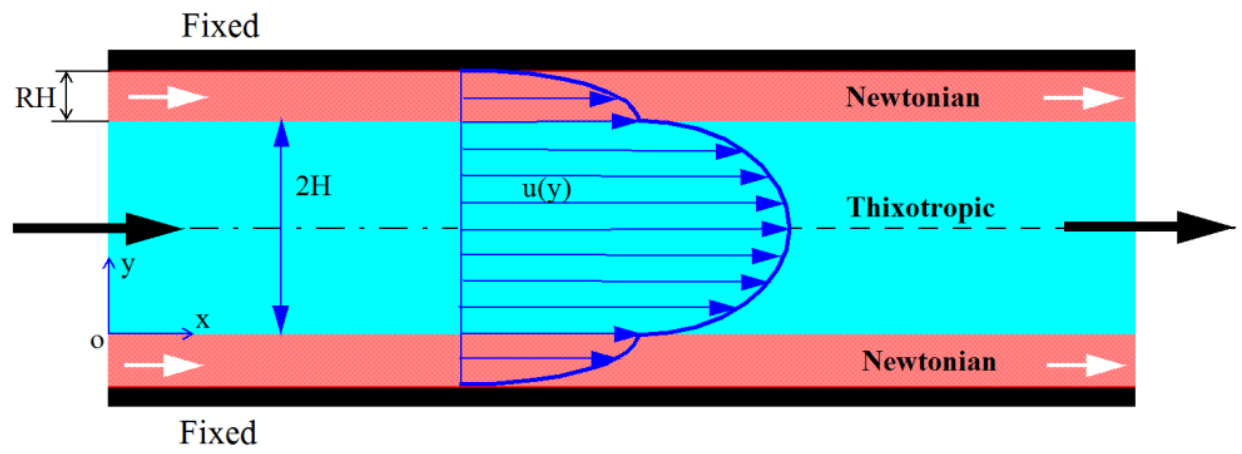

Fig. 1. Triple-layered channel flow considered for the analysis in this work.

laminated sheets. It can also be considered as twodimensional representation of the core-annular flow of heavy oils in lubricated pipelines. Similarly, it can be used to model the flow of cementslurry/drilling-muds in the annulus between the borehole and the rock formation in drilling industry.

As can be seen in Fig. 1, the height of the core fluid (which is thixotropic) is $2 \mathrm{H}$, and the thickness of the upper and lower Newtonian fluid layers is equal to $\mathrm{RH}$, where $\mathrm{R}$ is a real number. The fluids are assumed to be incompressible and the flow is isothermal. No gravity effects are involved in the problem. The flow is typical of plane Poiseuille flow. The upper and lower plates are parallel, rigid and fixed. Cartesian coordinate system is to be used for the mathematical development with its origin located somewhere on the lower interface.

The width of the channel normal to the plane (i.e., in the z-direction) is assumed to be infinity such that it serves as the neutral direction. On the other hand, the length of the plates are large enough for the base-flow to be assumed fully-developed. (driven by the same negative pressure gradient, $G$, for all layers). It is the instability of this base-flow which we would like to study in the present work. To that end, we need equations governing the flow of each fluid in each layer. To achieve this goal, we have to start from the unsteady form of the Cauchy equations of motion because as soon as the baseflow becomes unstable, we will be dealing with an unsteady flow which, for ease of analysis, is assumed to be two-dimensional based on the Squire's theorem.

The equations governing the unsteady flow which ensues when the base-flow becomes unstable comprise the conservation of mass and the conservation of momentum for each fluid. For the upper and lower Newtonian fluid layers, the equations of motions are just the Navier-Stokes equations. For the core fluid, in principle, we have to start from the Cauchy equations of motion which involves the fluid's deviatoric stress tensor. In the present work, however, although the core fluid is non-Newtonian, it is an inelastic fluid with a viscosity which is both time- and shear-dependent. In other words, the core fluid belongs to the generalized Newtonian class of non-Newtonian fluids (GNF's) for which we can use the variableviscosity version of the Navier-Stokes equations as the equations of motion; that is:

$$
\begin{aligned}
& \rho \frac{\mathrm{Du}}{\mathrm{Dt}}=-\frac{\partial \mathrm{p}}{\partial \mathrm{x}}+\frac{\partial}{\partial \mathrm{x}}\left[\mu\left(2 \frac{\partial \mathrm{u}}{\partial \mathrm{x}}\right)\right]+\frac{\partial}{\partial \mathrm{y}}\left[\mu\left(\frac{\partial \mathrm{u}}{\partial \mathrm{y}}+\frac{\partial \mathrm{v}}{\partial \mathrm{x}}\right)\right] \\
& \rho \frac{\mathrm{Dv}}{\mathrm{Dt}}=-\frac{\partial \mathrm{p}}{\partial \mathrm{y}}+\frac{\partial}{\partial \mathrm{x}}\left[\mu\left(\frac{\partial \mathrm{u}}{\partial \mathrm{y}}+\frac{\partial \mathrm{v}}{\partial \mathrm{x}}\right)\right]+\frac{\partial}{\partial \mathrm{y}}\left[\mu\left(2 \frac{\partial \mathrm{v}}{\partial \mathrm{y}}\right)\right] \\
& \frac{\partial \mathrm{u}}{\partial \mathrm{x}}+\frac{\partial \mathrm{v}}{\partial \mathrm{y}}=0
\end{aligned}
$$

where $\mu(t, \dot{\gamma})$ is the viscosity of the core fluid, $\rho$ denotes its density, $\mathrm{p}$ is the isotropic pressure, and $(\mathrm{u}, \mathrm{v})$ are the velocity components in the core of the channel. Also, $x$ and $y$ are respectively the streamwise and cross-stream coordinates with D/Dt serving as the material derivative. The thixotropic model used in this work is the Moore model (Moore 1959; Cheng and Evans, 1965). The viscosity of the core fluid (shown by subscript "1") obeying this rheological model is linearly dependent on a "structural parameter", $\lambda$, as shown below:

$\mu_{1}=\mu_{\infty}(1+\alpha \lambda)$,

where the "structural parameter" is time- and sheardependent scalar ranging between 0 and 1 with the former referring to a fully-broken-down structure and the latter to a fully-structured material. In this relationship, $\alpha=\left(\mu_{0}-\mu_{\infty}\right) / \mu_{\infty}$ is the viscositydifference ratio, with $\mu_{0}$ and $\mu_{\infty}$ being the zeroshear and infinite-shear viscosities of the material (which correspond to $\lambda=1$ and $\lambda=0$, respectively). It is worth-mentioning that, in practice, the fluid may settle at an equilibrium viscosity somewhere between these two limiting values. (Under equilibrium, the number of rebuilt microstructures balances the number of brokendown microstructures.) It is also to be noted that, the Moore model can represent Newtonian fluids with a viscosity equal to $\mu_{\infty}$ by simply setting $\alpha=0$. There are many structural thixotropic models in the literature. A key feature of the Moore model is that in this model $\lambda$ satisfies the kinetic equation:

$\frac{\partial \lambda}{\partial \mathrm{t}}+\mathrm{u}_{1} \frac{\partial \lambda}{\partial \mathrm{x}}+\mathrm{v}_{1} \frac{\partial \lambda}{\partial \mathrm{y}}=\mathrm{a}(1-\lambda)-\mathrm{b} \lambda \dot{\gamma}_{1}$ 
where "a" and "b" are material properties controlling the rate of structure rebuild (through Brownian motions) and structure breakdown (through shear deformation) - they are both positive numbers with "a" having the dimension of inverse time while " $b$ " is dimensionless. In Eq. 5, $\dot{\gamma}=\sqrt{\left|\mathrm{II}_{2 \mathbf{d}}\right|}$ is the effective shear rate with $\mathrm{II}_{2 \mathbf{d}}$ being the second invariant of the rate-of-deformation tensor (2d). In a two-dimensional core flow, $\dot{\gamma}$ can be related to the velocity field as:

$\dot{\gamma}_{1}=\sqrt{2\left(\frac{\partial \mathrm{u}_{1}}{\partial \mathrm{x}}\right)^{2}+2\left(\frac{\partial \mathrm{v}_{1}}{\partial \mathrm{y}}\right)^{2}+\left(\frac{\partial \mathrm{u}_{1}}{\partial \mathrm{y}}+\frac{\partial \mathrm{v}_{1}}{\partial \mathrm{x}}\right)^{2}}$

where, again, subscript "1" means the core fluid. Figure 2 shows the time-evolution of the Moore fluid's viscosity at different shear rates and different b/a ratios in steady shear for a typical $\mu_{0}=2, \mu_{\infty}=1$.

These results clearly demonstrate the simultaneous shear-thinning and thixotropic nature of Moore fluids. That is, for a given b/a the viscosity of a Moore fluid drops when shear rate is increased rather slowly with time until equilibrium is reached somewhere between $\mu_{0}$ and $\mu_{\infty}$. Similarly, at a given shear rate, the viscosity of Moore fluid is reduced as time elapses but at a rate which is controlled by b/a until equilibrium is reached. Interestingly, the time needed by the fluid to reach the equilibrium state becomes shorter and shorter the higher the shear rate or the larger the b/a ratio (see Fig. 2). In fact, based on this figure, the equilibrium viscosity becomes smaller the larger the shear rate or the larger the b/a ratio. And the equilibrium viscosity becomes closer to the infinite-shear viscosity $\left(\mu_{\infty}=1\right)$ when the shear rate and/or the b/a ratio is increased. Obviously, as earlier mentioned, Moore fluids exhibit shear-thinning in addition to exhibiting thixotropic behavior. It is also evident that the thixotropic behavior of this fluid model is controlled by the b/a ratio which can be interpreted as the "characteristic time" of the fluid.

\subsection{Dimensionless Numbers}

To work with dimensionless numbers, we can use $\mathrm{H}$ as the length scale, $\mathrm{H}^{2} \mathrm{G} / \mu_{\infty}$ as the velocity scale, $\mathrm{HG}$ as the pressure scale, and $\mu_{\infty} / \mathrm{HG}$ as the time scale. Obviously (with the flow being driven by pressure-gradient) the time scale can be interpreted as the "characteristic time of the flow". As earlier mentioned, the ratio b/a can be interpreted as the "characteristic time of the fluid". Their ratio is a dimensionless number referred to as the thixotropy number $\zeta$ :

$$
\zeta=\frac{\mathrm{HG}(\mathrm{b} / \mathrm{a})}{\mu_{\infty}}
$$

In practice, the competition between these two characteristic times determines the severity of any thixotropic effect. Fluids for which the thixotropy number is small need more time to reach a steady state, and so can be viewed as strongly thixotropic.
On the other hand, fluids having a large thixotropy number are weakly thixotropic but highly shearthinning. These two limiting behavior can best be seen in Fig. 3 which shows the effect of the thixotropy number on the structural parameter (which is linearly related to viscosity; see Eq. 4).
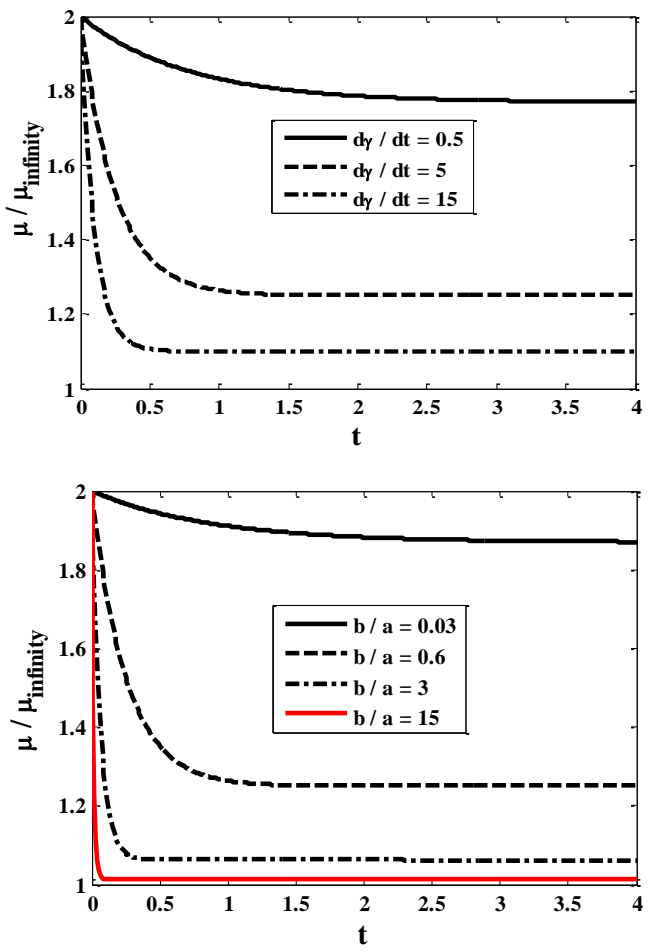

Fig. 2. Variation of the dimensionless viscosity of a Moore fluid with time in simple shear: (top plot) effect of the shear rate $(b / a=0.6)$, (bottom plot) effect of the $b / a$ ratio $(\dot{\gamma}=5)$.

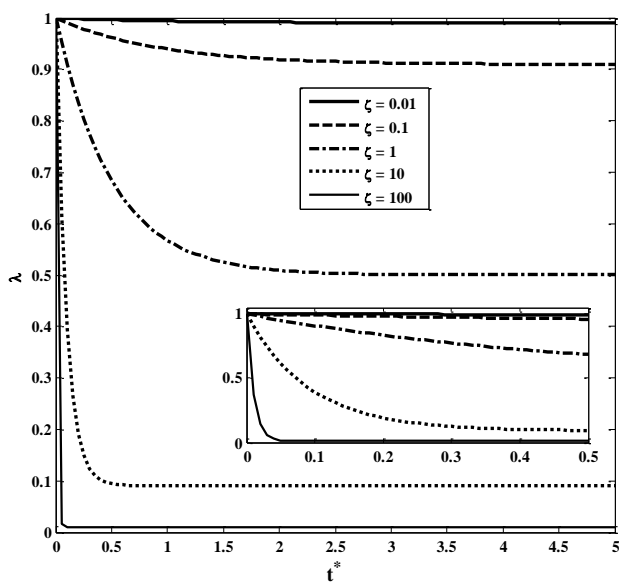

Fig. 3. Variation of structural parameter with time for different thixotropy numbers for a Moore fluid in steady shear flow $(\dot{\gamma}=5)$.

Another important dimensionless number involved in our fluid mechanics problem is the Reynolds number which is defined as:

$\operatorname{Re}_{\mathrm{i}}=\frac{\rho_{\mathrm{i}} \mathrm{H}^{3} \mathrm{G}}{\mu_{\infty}^{2}}$ 
where $\mathrm{i}=1$ stands for the core fluid and $\mathrm{i}=2$ for Newtonian layers. In dimensionless form, Eq. 5 becomes,

$\Phi\left(\frac{\partial \lambda}{\partial \mathrm{t}}+\mathrm{u}_{1} \frac{\partial \lambda}{\partial \mathrm{x}}+\mathrm{v}_{1} \frac{\partial \lambda}{\partial \mathrm{y}}\right)=(1-\lambda)-\zeta \lambda \dot{\gamma}_{1}$

where $\Phi=\mathrm{HG} / \mathrm{a} \mu_{\infty}$ is the dimensionless rebuild number. It should be noted that everything in this equation is now dimensionless. We can also define the cross-viscosity ratio, $C_{\mu}=\mu_{2} / \mu_{\infty}$ (where $\mu_{2}$ is the viscosity of the Newtonian fluids shown by subscript 2) as an important dimensionless number which might affect the instability picture. As the last dimensionless number involved in our problem, we can use $\mathrm{R}$ as the dimensionless thickness ratio (see Fig. 1). So, in general, the problem at hand is controlled by four dimensionless numbers: $\operatorname{Re}, \zeta$, $\mathrm{C}_{\mu}$, and $\mathrm{R}$.

\subsection{Boundary Conditions}

The boundary conditions needed to solve the equations of motion (say, after the interface becomes unstable) depends on the layer under consideration. For the Newtonian layers, we can impose the no-slip and no-penetration conditions at the walls. At the interface between the core fluid and the Newtonian fluid layers we can impose the continuity of velocity and (tangential/normal) stresses. We can also impose the symmetry condition for the thixotropic fluid at the centerline of the channel. The symmetry of the flow configuration also entitles us to consider only half of the channel. Focusing on just the lower half, at the middle of the channel $(\mathrm{y}=1)$ we have:

$$
\frac{\mathrm{du}_{1}}{\mathrm{dy}}=0 ; \mathrm{v}_{1}=0 ; \lambda=1,
$$

where the boundary condition on $\lambda$ is based on the notion that along the centerline of the channel, shear stress is equal to zero and microstructures are not broken down. At the wall $(\mathrm{y}=-\mathrm{R})$, based on no-slip and no-penetration boundary conditions, we have:

$\mathrm{u}_{2}=0=\mathrm{v}_{2}$.

At the interface $(y=0)$, the kinematic/dynamic boundary conditions are:

$$
@ \mathrm{y}=0: \mathrm{u}_{1}=\mathrm{u}_{2} ; \mathrm{p}_{1}=\mathrm{p}_{2} ; \tau_{1}=\tau_{2} \text {. }
$$

The shear stress boundary condition leads to the following equation:

$\left.\left(1+\left.\alpha \lambda\right|_{y=0}\right) \frac{d u_{1}}{d y}\right|_{y=0}=\left.C_{\mu} \frac{d u_{2}}{d y}\right|_{y=0}$.

This equation involves the value of $\lambda$ at $y=0$ which is not known. To circumvent this problem, using Eq. 5 , we can related $\lambda(0)$ to the velocity gradient at this location. Thus, Eq. 11 becomes:

$$
0=\mathrm{a}(1-\lambda)-\mathrm{b} \lambda \dot{\gamma}_{1} \Rightarrow \lambda(0)=\frac{\mathrm{a}}{\mathrm{a}-\mathrm{b} \dot{\gamma}_{1}(0)} .
$$

So, in dimensionless form, Eq. 11a becomes:

$$
\Rightarrow\left(1+\frac{\alpha}{\left(1+\left.\zeta \frac{d u_{1}}{d y}\right|_{y=0}\right)}\right) \times\left.\frac{\mathrm{du}_{1}}{\mathrm{dy}}\right|_{\mathrm{y}=0}=\left.\mathrm{C}_{\mu} \frac{\mathrm{du}_{2}}{\mathrm{dy}}\right|_{\mathrm{y}=0},
$$

which, fortunately, involves just the velocity gradients at $y=0$. It needs to be mentioned that, the boundary conditions on $\lambda$ are in line with those proposed by Billingham and Ferguson (1993) in their investigating the pipe flow of Moore fluids. We also would like to stress that in the present study we have ignored surface tension effects simply because the working fluids have been assumed to be slightly miscible. This scenario can be encountered in coating plastic sheets. It is also the case with the cementing operation of oil wells where cement slurry and drilling muds are known to be miscible even though their mixing is so weak that, in practice, the mixing is just confined to a thin mixing layer near the interface during the solidification phase (Frigaard and Crawshaw, 1999). Obviously, surface tension effects should be taken into account for immiscible fluids, for example, when water (not kerosene) is used in core annular flow of heavy oils to lubricate the wall. But, even for immiscible fluids, surface tension cannot affect the base flow when the interface is flat, like that shown in Fig. 1. In fact, even for immiscible fluids, surface-tension effects enter the analysis as soon as the interface becomes wavy-shaped. And when it plays a role, its effect is confined to affecting short-wave modes where the radius of curvature is small. But not all systems are vulnerable to short waves. For systems which are vulnerable, surface tension is already known to stabilize wavenumbers larger than a cut-off value for unbounded flat interfaces (Bellman and Pennington, 1954). For a curved interface, such as that encountered in core-annular flow, its effect can be stabilizing or destabilizing depending on the curvatures of the two fluid layers (Wei, 2005).

\section{BASE FLOW}

A knowledge about the velocity profiles and structural parameter under steady conditions (the socalled base-flow) is needed in our linear stability analysis. The base-flow corresponds to fullydeveloped flow for each layer. For the upper and lower Newtonian layers the equations of motion (developed in the previous section) are reduced to:

$0=-\frac{\partial \mathrm{p}_{2}}{\partial \mathrm{x}}+\mathrm{C}_{\mu} \frac{\partial^{2} \mathrm{u}_{2}}{\partial \mathrm{y}^{2}}$

where $u_{2}(y)$ is the base-flow velocity profile for the fluid in the wall layers. For the core thixotropic fluid, the pertinent equations governing the base-flow are:

$0=-\frac{\partial \mathrm{p}_{1}}{\partial \mathrm{x}}+\frac{\partial}{\partial \mathrm{y}}\left[(1+\alpha \lambda) \frac{\partial \mathrm{u}_{1}}{\partial \mathrm{y}}\right]$ 


$$
0=(1-\lambda)-\zeta \lambda\left|\frac{\mathrm{du}_{1}}{\mathrm{dy}}\right|
$$

where $\mathrm{u}_{1}(\mathrm{y})$ is the core-flow velocity profile. Now, using the boundary conditions set forth above (see Eqs. 10-12), the base-flow velocity profiles in the Newtonian layers is easily obtained as:

$\mathrm{u}_{2}(\mathrm{y})=\frac{\mathrm{G}}{\mathrm{C}_{\mu}}(\mathrm{y}+\mathrm{R})\left(\frac{\mathrm{y}-\mathrm{R}}{2}-1\right)$.

For the core fluid we can easily show that we have:

$$
\begin{aligned}
& \mathrm{u}_{1}(\mathrm{y})=\frac{\mathrm{G}}{2}\left(\frac{\mathrm{y}^{2}}{2}-\mathrm{y}-\frac{\mathrm{R}^{2}+2 \mathrm{R}}{\mathrm{C}_{\mu}}\right)-\frac{(1+\alpha)}{2 \zeta} \mathrm{y}+ \\
& \sqrt{\mathrm{k}_{1} \mathrm{y}^{2}+\mathrm{k}_{2} \mathrm{y}+\mathrm{k}_{3}}\left(\frac{\mathrm{y}}{2}+\frac{\mathrm{k}_{2}}{4 \mathrm{k}_{1}}\right)+\frac{\left(\mathrm{k}_{1} \mathrm{k}_{3}-\frac{\mathrm{k}_{2}^{2}}{4}\right)}{2 \mathrm{k}_{1} \sqrt{\mathrm{k}_{1}}} \times \\
& \ln \left(\frac{2 \mathrm{k}_{1} \mathrm{y}+\mathrm{k}_{2}+2 \sqrt{\mathrm{k}_{1}} \sqrt{\mathrm{k}_{1} \mathrm{y}^{2}+\mathrm{k}_{2} \mathrm{y}+\mathrm{k}_{3}}}{\mathrm{k}_{2}+2 \sqrt{\mathrm{k}_{1} \mathrm{k}_{3}}}\right)- \\
& \sqrt{\mathrm{k}_{3}}\left(\frac{\mathrm{k}_{2}}{4 \mathrm{k}_{1}}\right),
\end{aligned}
$$

where we have:

$$
\begin{aligned}
& \mathrm{k}_{1}=\zeta^{2} \mathrm{G}^{2}, \\
& \mathrm{k}_{2}=2 \zeta \mathrm{G}(1-\alpha-\zeta \mathrm{G}), \\
& \mathrm{k}_{3}=(1+\alpha+\zeta \mathrm{G})^{2}-4 \zeta \mathrm{G} .
\end{aligned}
$$

The above analytical solutions has enabled us to easily investigate the effect of different parameters on the base-flow velocity profiles across the whole channel, $u(y)$. Figures 4-6 show typical results obtained for $G=-1$. The effect of cross-viscosity ratio on the base-flow velocity profile has been shown in Fig. 4. For a given zero-shear viscosity (say, the viscosity of water) an increase in this ratio means an increase in the viscosity of the fluid near the wall. And, the results shown in this figure suggests that it has a retarding effect on the core flow, as expected.

Figure 5 shows the effect of the thickness parameter $(\mathrm{R})$ on the base-flow velocity profile. Based on this figure, one can conclude that an increase in the thickness of the wall layer (for a given thickness of the core layer) increases the core velocity.

Figure 6 shows the effect of the thixotropy number on the velocity profile, $\mathrm{u}(\mathrm{y})$. As expected, the thixotropy number strongly affects the velocity profile in the core but not in the Newtonian layers. The strong effect of thixotropy number on the core fluid can be explained by its effect on the viscosity of the core fluid. With the flow occurring under steady conditions, variation in the viscosity in the $y$-direction for the core fluid is due to its shear- thinning behavior and the fact that shear stress varies linearly across the channel starting from zero at centerline.

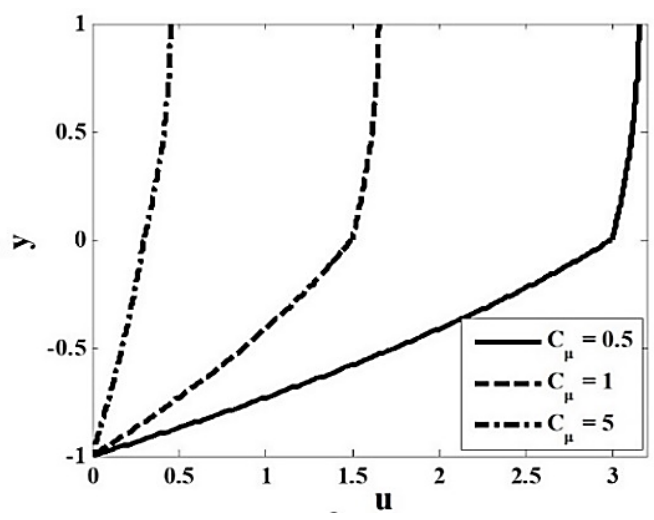

Fig. 4. Effects of the cross-viscosity ratio on the base-flow velocity profile $(\mathrm{R}=1, \alpha=2.5, \xi=0.5)$.

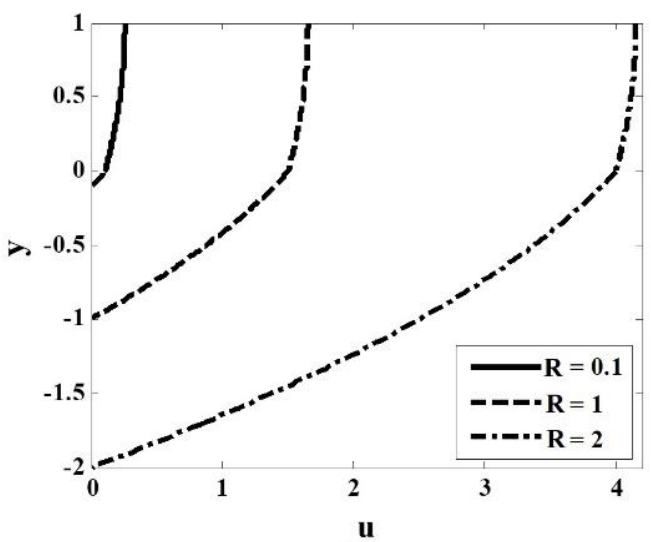

Fig. 5. Effects of the thickness parameter $(R)$ on the base-flow velocity profile $\left(\alpha=2.5, C_{\mu}=1, \zeta=0.5\right)$.

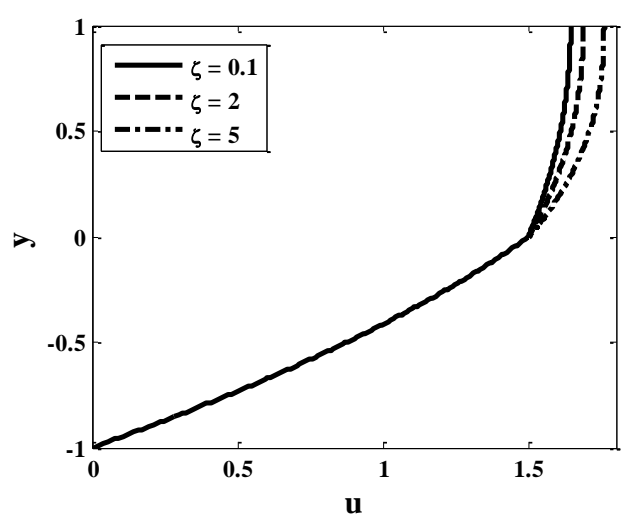

Fig. 6. Effect of the thixotropy number $(\zeta)$ on the base-flow velocity profile and viscosity variation $\left(\mathrm{R}=1, \alpha=2.5, \mathrm{C}_{\mu}=1\right)$.

Figure 7 shows the effect of viscosity-difference ratio on the velocity and viscosity profiles. As expected, this parameter strongly affects the velocity profile in the core but not in the Newtonian layers. 

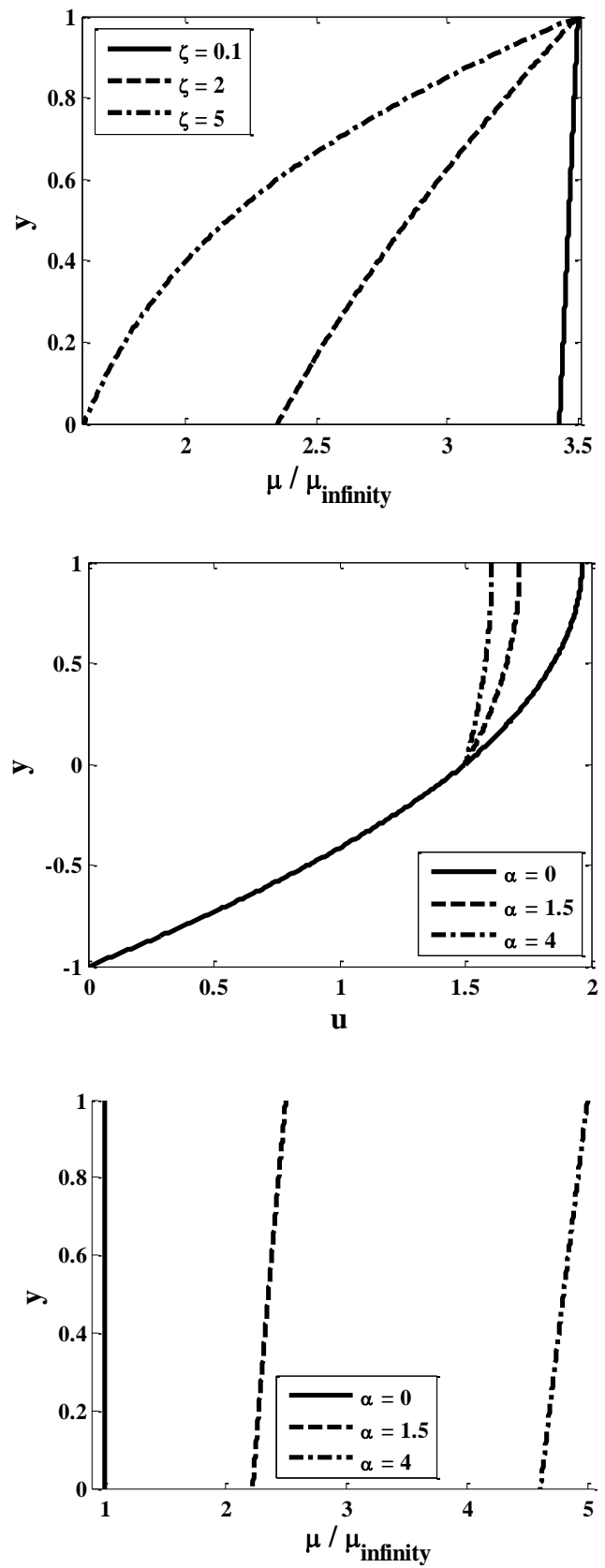

Fig. 7. Effect of viscosity-difference ratio $(\alpha)$ on base-flow velocity profile and viscosity variation.

$$
\left(\mathrm{R}=1, \zeta=0.5, \mathrm{C}_{\mu}=1\right) \text {. }
$$

The velocity profiles shown in Figs. 4-7 will be used in our stability analysis.

\section{Linear StabiLity ANALYSIS}

Having found the base-flow velocity profiles, we can proceed with perturb them by infinitesimally small disturbances and see if there exists any mode which can become unstable. To this end, the perturbed velocity and pressure fields are expressed in terms of normal modes as follows (Drazin, 1981):

$\mathrm{u}_{\mathrm{i}}(\mathrm{x}, \mathrm{y}, \mathrm{t})=\overline{\mathrm{u}}_{\mathrm{i}}(\mathrm{y})+\tilde{\mathrm{u}}_{\mathrm{i}}(\mathrm{y}) \mathrm{e}^{\mathrm{i} \kappa \mathrm{x}+\omega \mathrm{t}}$

$$
\begin{aligned}
& \mathrm{v}_{\mathrm{i}}(\mathrm{x}, \mathrm{y}, \mathrm{t})=\overline{\mathrm{v}}_{\mathrm{i}}(\mathrm{y})+\tilde{\mathrm{v}}_{\mathrm{i}}(\mathrm{y}) \mathrm{e}^{\mathrm{i} \kappa \mathrm{x}+\omega \mathrm{t}}, \\
& \mathrm{p}_{\mathrm{i}}(\mathrm{x}, \mathrm{y}, \mathrm{t})=\overline{\mathrm{p}}_{\mathrm{i}}(\mathrm{y})+\tilde{\mathrm{p}}_{\mathrm{i}}(\mathrm{y}) \mathrm{e}^{\mathrm{i} \kappa \mathrm{x}+\omega \mathrm{t}}, \\
& \lambda(\mathrm{x}, \mathrm{y}, \mathrm{t})=\bar{\lambda}(\mathrm{y})+\tilde{\lambda}(\mathrm{y}) \mathrm{e}^{\mathrm{i} \kappa \mathrm{x}+\omega \mathrm{t}} .
\end{aligned}
$$

where "bar" means "base flow" and "tilde" means "perturbation" variables. In these equations the subscript " $i$ " can be 1 representing the core fluid and 2 representing the wall layer. After substituting perturbed velocity, pressure, and structuralparameter fields into the equations of motion, we can neglect nonlinear perturbation terms. Having eliminated pressure (through cross-differentiation) and then subtracting the base-flow we end up with the following equation for the Newtonian layers:

$$
\frac{\mathrm{d}^{4} \tilde{\mathbf{v}}_{2}}{d \mathrm{y}^{4}}-2 \kappa^{2} \frac{\mathrm{d}^{2} \tilde{\mathrm{v}}_{2}}{\mathrm{dy}^{2}}+\kappa^{4} \tilde{\mathrm{v}}_{2}+\operatorname{Re}_{2}\left[\begin{array}{l}
\mathrm{i} \kappa \frac{\mathrm{d}^{2} \overline{\mathbf{u}}_{2}}{\mathrm{dy} \mathrm{v}^{2}} \tilde{\mathrm{v}}_{2}+\left(\omega+\mathrm{i} \kappa \overline{\mathrm{u}}_{2}(\mathrm{y})\right) \times \\
\left(\kappa^{2} \tilde{\mathrm{v}}_{2}-\frac{\mathrm{d}^{2} \tilde{\mathrm{v}}_{2}}{\mathrm{dy}^{2}}\right)+
\end{array}\right]=0
$$

which is the classic Orr-Sommerfeld equation although in the current study it must be solved subject to a different boundary condition (i.e., the interfacial boundary condition). Similarly, for the thixotropic fluid flowing through the core, we end up with the following equation:

$$
\begin{aligned}
& \mathrm{c}_{1}(\mathrm{y}) \frac{\mathrm{d}^{4} \tilde{\mathrm{v}}_{1}}{\mathrm{dy}^{4}}+\mathrm{c}_{2}(\mathrm{y}) \frac{\mathrm{d}^{3} \tilde{\mathrm{v}}_{1}}{\mathrm{dy}^{3}}+\mathrm{c}_{3}(\mathrm{y}) \frac{\mathrm{d}^{2} \tilde{\mathrm{v}}_{1}}{\mathrm{dy}^{2}}+\mathrm{c}_{4}(\mathrm{y}) \frac{\mathrm{d} \tilde{\mathrm{v}}_{1}}{\mathrm{dy}}+ \\
& \mathrm{c}_{5}(\mathrm{y}) \tilde{\mathrm{v}}_{1}+\mathrm{c}_{6}(\mathrm{y}) \frac{\mathrm{d}^{2} \tilde{\lambda}}{\mathrm{dy}^{2}}+\mathrm{c}_{7}(\mathrm{y}) \frac{\mathrm{d} \tilde{\lambda}}{\mathrm{dy}}+\mathrm{c}_{8}(\mathrm{y}) \tilde{\lambda}=0
\end{aligned}
$$

where we have:

$$
\begin{aligned}
& \mathrm{c}_{1}(\mathrm{y})=\frac{1}{\kappa^{2}(1+\alpha \bar{\lambda}(\mathrm{y}))^{2}} \\
& \mathrm{c}_{2}(\mathrm{y})=-\frac{4 \alpha}{\kappa^{2}(1+\alpha \bar{\lambda})^{3}} \frac{\mathrm{d} \bar{\lambda}}{\mathrm{dy}} \\
& \mathrm{c}_{3}(\mathrm{y})=\frac{6 \alpha^{2}}{\kappa^{2}(1+\alpha \bar{\lambda}(\mathrm{y}))^{4}}\left(\frac{\mathrm{d} \bar{\lambda}}{\mathrm{dy}}\right)^{2}-\frac{\mathrm{Re}}{\kappa^{2}}\left(\omega+\mathrm{i} \kappa \overline{\mathrm{u}}_{1}(\mathrm{y})\right) \\
& -\frac{2 \alpha}{\kappa^{2}(1+\alpha \bar{\lambda}(\mathrm{y}))^{3} \frac{\mathrm{d}^{2} \bar{\lambda}}{\mathrm{dy}}}-\frac{2}{(1+\alpha \bar{\lambda}(\mathrm{y}))^{3}} \\
& \mathrm{c}_{4}(\mathrm{y})=\frac{4 \alpha}{(1+\alpha \bar{\lambda}(\mathrm{y}))^{3}} \frac{\mathrm{d} \bar{\lambda}}{\mathrm{dy}} \\
& \mathrm{c}_{5}(\mathrm{y})=\frac{\kappa^{2}}{(1+\alpha \bar{\lambda}(\mathrm{y}))^{2}}+\operatorname{Re}\left(+\mathrm{i} \kappa \overline{\mathrm{u}}_{1}(\mathrm{y})-\frac{1}{\mathrm{i \kappa}} \frac{\mathrm{d} \overline{\mathrm{u}}_{1}}{\mathrm{dy}}+\omega\right)+ \\
& \frac{6 \alpha^{2}}{(1+\alpha \bar{\lambda}(\mathrm{y}))^{4}}\left(\frac{\mathrm{d} \bar{\lambda}}{\mathrm{dy}}\right)^{2}-\frac{2 \alpha}{(1+\alpha \bar{\lambda}(\mathrm{y}))^{3}} \frac{\mathrm{d}^{2} \bar{\lambda}}{\mathrm{dy} y^{2}}
\end{aligned}
$$




$$
\begin{aligned}
& c_{6}(y)=\frac{2 i}{\kappa} \frac{\alpha}{(1+\alpha \bar{\lambda}(y))^{3}} \frac{d \bar{u}_{1}}{d y} \\
& c_{7}(y)=-\frac{12 i}{\kappa} \frac{\alpha^{2}}{(1+\alpha \bar{\lambda}(y))^{4}} \frac{d \bar{u}_{1}}{d y} \frac{d \bar{\lambda}}{d y}+ \\
& \frac{4 i}{\kappa} \frac{\alpha}{(1+\alpha \bar{\lambda}(y))^{3}} \frac{d^{2} \bar{u}_{1}}{d y^{2}} \\
& c_{8}(y)=\frac{24 i}{\kappa} \frac{\alpha^{3}}{(1+\alpha \bar{\lambda}(y))^{5}} \frac{d \bar{u}_{1}}{d y} \frac{d \bar{\lambda}}{d y}-\frac{6 i}{\kappa} \frac{\alpha^{2}}{(1+\alpha \bar{\lambda}(y))^{4}} \frac{d \bar{u}_{1}}{d y} \frac{d^{2} \bar{\lambda}}{d y^{2}}- \\
& \frac{12 i}{\kappa} \frac{\alpha^{2}}{(1+\alpha \bar{\lambda}(y))^{4}} \frac{d^{2} \bar{u}_{1}}{d y^{2}} \frac{d \bar{\lambda}}{d y}+\frac{2 i}{\kappa} \frac{\alpha}{(1+\alpha \bar{\lambda}(y))^{3}}\left(\frac{d^{3} \bar{u}_{1}}{d y^{3}}+\kappa^{2} \frac{d \bar{u}_{1}}{d y}\right)
\end{aligned}
$$

For the structural parameter $(\lambda)$ we have:

$$
a_{1}(y) \frac{d^{2} \tilde{v}_{1}}{d y^{2}}+a_{2}(y) \tilde{v}_{1}+a_{3}(y) \tilde{\lambda}=0
$$

where,

$$
\begin{aligned}
& \mathrm{a}_{1}(\mathrm{y})=-\frac{\zeta}{\mathrm{i} \kappa} \operatorname{sgn}\left(\left|\frac{\mathrm{d} \overline{\mathrm{u}}_{1}}{\mathrm{dy}}\right|\right) \bar{\lambda}(\mathrm{y}) \\
& \mathrm{a}_{2}(\mathrm{y})=\Phi \frac{\mathrm{d} \bar{\lambda}}{\mathrm{dy}}+\mathrm{i} \zeta \operatorname{sgn}\left(\left|\frac{\mathrm{d} \overline{\mathrm{u}}_{1}}{\mathrm{dy}}\right|\right) \bar{\lambda}(\mathrm{y}) \\
& \mathrm{a}_{3}(\mathrm{y})=1+\Phi \omega+\Phi i \kappa \overline{\mathrm{u}}_{1}(\mathrm{y})+\zeta\left|\frac{\mathrm{d} \overline{\mathrm{u}}_{1}}{\mathrm{dy}}\right|
\end{aligned}
$$

As to boundary conditions, obviously, the two Newtonian fluid layers should satisfy the no-slip and no-penetration conditions at their respective walls. They should also satisfy the interfacial kinematic and dynamic boundary conditions in contact with the Moore fluid. By focusing only the symmetric modes in our instability analysis, the core fluid should satisfy the symmetry conditions at the center of the channel. Thus, at the center line of channel and also the lower wall we should have:

$$
\begin{aligned}
& \left.\tilde{\mathrm{v}}_{1}\right|_{\mathrm{y}=1}=0 ;\left.\frac{\mathrm{d} \tilde{\mathrm{u}}_{1}}{\mathrm{dy}}\right|_{\mathrm{y}=1}=0 ;\left.\frac{\mathrm{d}^{2} \tilde{\mathrm{v}}_{1}}{\mathrm{dy}^{2}}\right|_{\mathrm{y}=1}=0,\left.\tilde{\lambda}_{1}\right|_{\mathrm{y}=1}=0 . \\
& \left.\tilde{\mathrm{v}}_{2}\right|_{\mathrm{y}=-\mathrm{R}} ;\left.\tilde{\mathrm{u}}_{2}\right|_{\mathrm{y}=-\mathrm{R}}=0 ;\left.\frac{\mathrm{d} \tilde{\mathrm{v}}_{2}}{\mathrm{dy}}\right|_{\mathrm{y}=-\mathrm{R}}=0,
\end{aligned}
$$

At the interface we have:

$$
\begin{aligned}
& \left.\tilde{\mathrm{u}}_{1}\right|_{\mathrm{y}=0}+\left.\frac{\mathrm{d} \overline{\mathrm{u}}_{1}}{\mathrm{dy}}\right|_{\mathrm{y}=0} \delta-\left(\left.\tilde{\mathrm{u}}_{2}\right|_{\mathrm{y}=0}+\left.\frac{\mathrm{d} \overline{\mathrm{u}}_{2}}{\mathrm{dy}}\right|_{y=0} \delta\right)=0 \\
& \left.\tilde{\mathrm{v}}_{1}\right|_{\mathrm{y}=0}-\left.\tilde{\mathrm{v}}_{2}\right|_{\mathrm{y}=0}=0 ; \\
& \left.\tilde{\tau}_{\mathrm{xy}, 1}\right|_{y=0}-\left.\tilde{\tau}_{\mathrm{xy}, 2}\right|_{y=0}+\left.\delta\left(\frac{\mathrm{d} \bar{\tau}_{\mathrm{xy}, 1}}{\mathrm{dy}}-\frac{\mathrm{d} \bar{\tau}_{\mathrm{xy}, 2}}{\mathrm{dy}}\right)\right|_{y=0}=0
\end{aligned}
$$

$$
\begin{aligned}
& \left.\left(\tilde{\mathrm{p}}_{2}-\tilde{\mathrm{p}}_{1}+\tilde{\tau}_{\mathrm{yy}, 1}\right)\right|_{\mathrm{y}=0}-\left.\tilde{\tau}_{\mathrm{yy}, 2}\right|_{\mathrm{y}=0}+ \\
& \left.\operatorname{i\kappa \delta }\left(\bar{\tau}_{\mathrm{xy}, 2}-\bar{\tau}_{\mathrm{xy}, 1}\right)\right|_{\mathrm{y}=0}=0,
\end{aligned}
$$

where $\delta$ is the vertical displacement of the perturbed interface which can be related to the normal perturbed velocity at the interface by the following kinematic condition:

$\frac{\partial \delta}{\partial \mathrm{t}}+\left.\overline{\mathrm{u}}_{1}\right|_{\mathrm{y}=0}\left(\frac{\partial \delta}{\partial \mathrm{x}}\right)=\left.\mathrm{v}_{1}^{\prime}\right|_{\mathrm{y}=0}$

In the next section, we discuss the numerical method for solving the instability equations.

\section{NUMERICAL METHOD}

Equations 20-22 can be solved numerically using the pseudo-spectral colocation method. To that end, all variables are expanded in a series consisting Chebyshev polynomials. Since Chebyshev polynomials are defined in the range of $[-1,1]$, we have to map the physical domain onto this range. To achieve this goal, we substitute:

$\mathrm{Y}=2 \mathrm{y}-1 ; 0 \leq \mathrm{y} \leq 1$,

$\mathrm{W}=\frac{2 \mathrm{y}+\mathrm{R}}{\mathrm{R}} ;-\mathrm{R} \leq \mathrm{y} \leq 0$.

The disturbance variables are then expressed as:

$\tilde{\mathrm{v}}_{1}=\sum_{\mathrm{n}=1}^{\mathrm{N}} \mathrm{a}_{\mathrm{n}} \mathrm{T}_{\mathrm{n}}(\mathrm{Y}), \tilde{\lambda}=\sum_{\mathrm{n}=1}^{\mathrm{N}} \mathrm{c}_{\mathrm{n}} \mathrm{T}_{\mathrm{n}}(\mathrm{Y}), \tilde{\mathrm{v}}_{2}=\sum_{\mathrm{n}=1}^{\mathrm{N}} \mathrm{b}_{\mathrm{n}} \mathrm{T}_{\mathrm{n}}(\mathrm{W})$

where $T_{n}(X)=\cos (n \cdot \arccos (X))$ are Chebyshev polynomials. After substituting equations (26) into Eqs. (20-22) and the boundary conditions (23-a,b) an eigenvalue problem is obtained in the form of $A X=\omega B X$ in which $\omega$ is the eigenvalue and $\mathrm{X}=\left[\mathrm{a}_{1}, \ldots, \mathrm{a}_{\mathrm{n}} ; \mathrm{b}_{1}, \ldots, \mathrm{b}_{\mathrm{n}} ; \mathrm{c}_{1}, \ldots, \mathrm{c}_{\mathrm{n}}\right]$ is the eigenvector. The eigenvalues, in general, are complex numbers. In our temporal stability analysis (where the wavenumber, $\kappa$, is a real number) if the real part of an eigenvalue is positive, the disturbance grows exponentially with time and the mode corresponding to this eigenvalue is unstable. In practice, however, we only have to look for neutral instability curve for which the real part of the eigenvalue is equal to zero. In pseudo-spectral collocation method, for each wave number, a spectrum of eigenvalues is obtained from which we can find the one which lies on the neutral stability curve. After doing this over a wide range of wavenumbers, the neutral curve is obtained from which we can find the critical Reynolds number and critical wavenumber. To verify the MATLAB. code developed in this work, we have tried to recover the critical Reynolds number for classic plane Poiseuille flow of a single-phase Newtonian fluid. The critical Reynolds number and wave number found in this benchmark are 5772.2214 and 1.0054, respectively, are virtually the same as those reported 
in the literature (Drazin, 1981). It is worth mentioning that to verify the code we had to use 150 Chebyshev terms to ensure grid-independent results.

\section{RESUlts AND DISCUSSION}

In this section, we present our numerical results addressing the effect of core fluid's rheology (as denoted by the thixotropy number and viscositydifference ratio) on the critical Reynolds number. We are also interested in the effect of cross-viscosity ratio and thickness of the wall layer on the instability picture. With no loss of generality, we fix $G=-1$ and $\Phi=1$. Also, unless otherwise stated, the density ratio of the two fluids is set equal to one.

Figure 8 shows the effect of the thixotropy number on the neutral stability curve. As can be seen in this figure, an increase in the thixotropy number from 0.5 to 1.5 destabilizes the flow. As earlier mentioned, fluids with a smaller thixotropy number exhibit stronger thixotropic effects. In contrast, those having a larger thixotropy number exhibit stronger shearthinning effects. One can therefore conclude that thixotropy has a stabilizing effect on the interfacial stability with the effect of shear-thinning being destabilizing on the interfacial modes. The destabilizing effect of shear-thinning has previously been reported by Su and Khomami (1991). But, this is the first time that the stabilizing effect of thixotropy on core-annular flow is being reported.

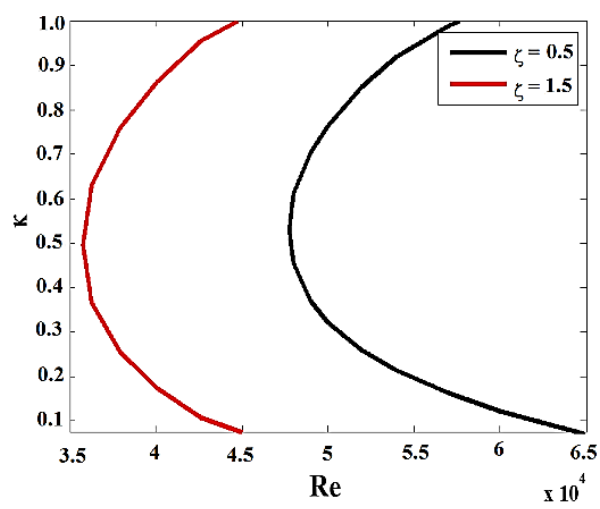

Fig. 8. Effect of the thixotropy number on the neutral stability curve $\left(R=1, \alpha=1.5, C_{\mu}=1\right)$.

The destabilizing effect of thixotropy number can better be seen in Fig. 9 which shows variation of the critical Reynolds number and wave number at two different thixotropy numbers over a broad range of cross-viscosity ratios. This figure shows that, for Moore fluids, there exists a threshold cross-viscosity at which the flow is at its most stable situation. This should be contrasted with Newtonian/Newtonian scenario where an increase in this ration has a monotonically destabilizing effect. The threshold is reduced when the thixotropy number is increased. Interestingly, the mode corresponding to the threshold has the smallest wavenumber (see Fig. 9). The results in this figure suggest that the interface can be stabilized if instead of water use can be made of more viscous Newtonian fluids although this increases the pressure drop.
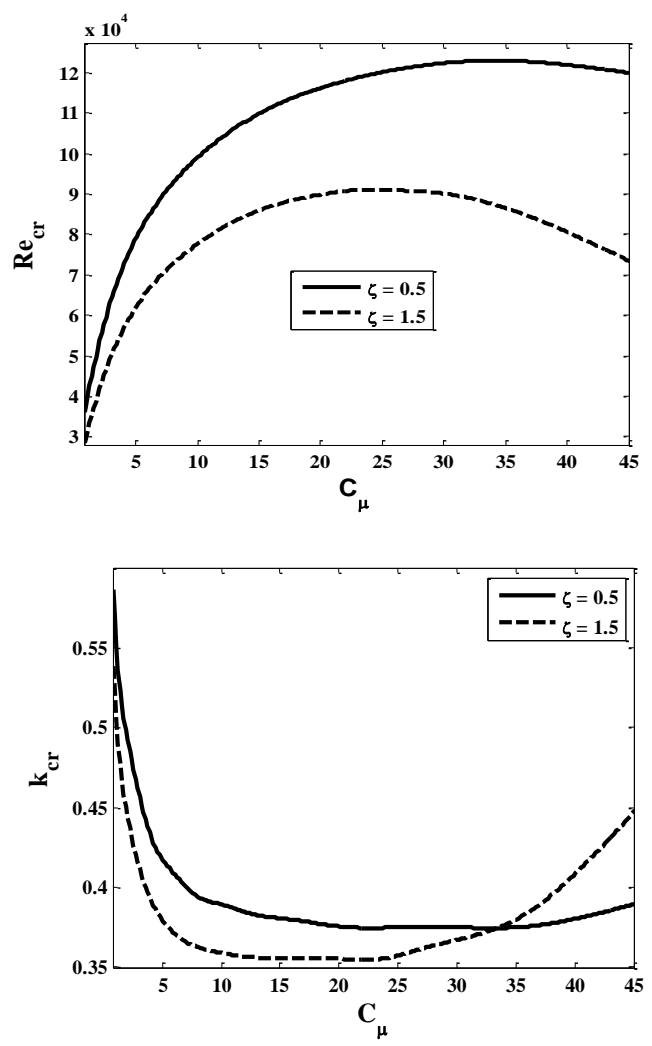

Fig. 9. Effect of the cross-viscosity ratio on the neutral stability curve $(R=1, \alpha=1.5)$.

Figure 10 shows the effect of the viscosity-difference ratio $(\alpha)$ on the neutral stability curve. This figure shows that an increase in $\alpha$ stabilizes the interface. This is because, for a given $\mu_{\infty}$, an increase in $\alpha$ means an increase in $\mu_{0}$. An increase in $\alpha$ makes the viscosity at the two sides of the interface closer to each other lowering the likelihood of the interface instability, and this explains why the interface becomes more stable when $\alpha$ is increased.

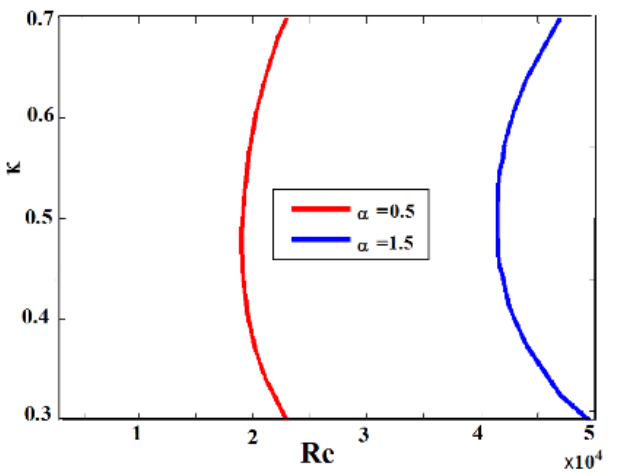

Fig. 10. Effect of the viscosity-difference ratio on the neutral stability curve

$\left(\mathrm{R}=1, \xi=0.5, \mathrm{C}_{\mu}=1\right)$.

Figure 11 shows the effect of cross-viscosity ratio on the variation of critical Reynolds number and critical 
wavenumber at several viscosity-difference ratios. Again, a threshold cross-viscosity ratio appears to exist at which the flow is at its most stable situation.
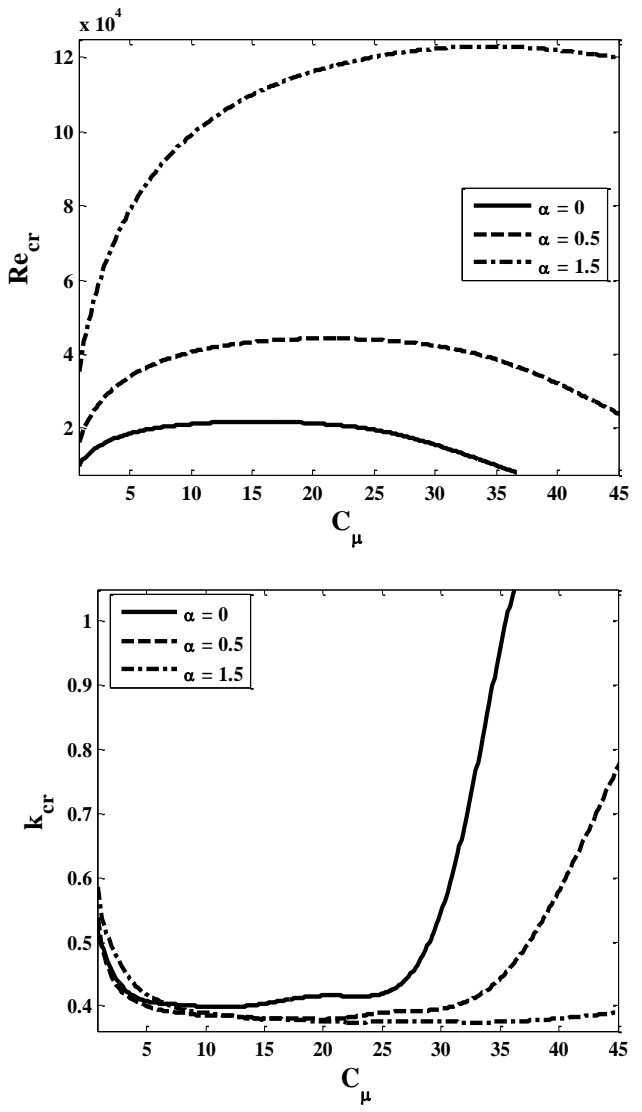

Fig. 11. Effect of the cross-viscosity ratio on the neutral stability curve $(R=1, \xi=0.5)$.

The results presented in the Fig. 9 and Fig. 11 show that the critical wavenumber is very small (say, of the order of $10^{-1}$ ) which means that the wavelength of the unstable modes is, at least, of the same order as $\mathrm{H}$. And this justifies ignoring the effect of surface tension from the analysis although that assumption was made right from beginning to screen out the complicating effects of surface tension from the analysis thereby enabling us to focus on just the role played by the rheology of the core fluid on the instability picture. (It can be shown that the critical wave numbers correspond to the interfacial modes, i.e., modes for which the perturbed velocities vary abruptly near $y=0$.) To interpret the role played by the rheological properties of the Moore fluid on the stability of the interface, it should be noted that with the density ratio being equal to one in these figures (i.e., with no gravity effects involved) the instability of the interface is only due to the viscosity mismatch at the interface although the role played by the thixotropy number (say, b/a ratio) can complicate the picture. For example, for the case in which the viscosity of the annular flow is equal to the infiniteshear viscosity of the core fluid (i.e., for $C_{\mu}=1$ ) the viscosity mismatch at $\mathrm{t}=0$ at the interface $(\mathrm{y}=0)$ is actually equal to $\Delta \mu=\mu_{0}-\mu_{\infty}$. But, depending on the b/a ratio in the Moore model, the viscosity of the core fluid is decreased at the early stages of instability (see Figs. 3 and4), and this lowers the viscosity mismatch giving rise to the stabilizing effect of the b/a ratio (i.e., the thixotropic time constant) of the fluid.

At this stage, we would like to stress that although the main objective of the present work has been to investigate the effect of core fluid's rheology on the instability of core-annular flow, for curiosity, we have decided to investigate the effect of thickness ratio $(\mathrm{R})$ and also density ratio $(\mathrm{Cr})$ on the instability picture. Figure 12 shows variation of the critical Reynolds number with thickness ratio for three different cross-viscosity ratios. Interestingly, for any cross-viscosity ratio, there appears to exist two extremum one corresponding to the most stable case and the other one to the least stable case although the wave numbers corresponding to these two cases are quite different (see Fig. 13).

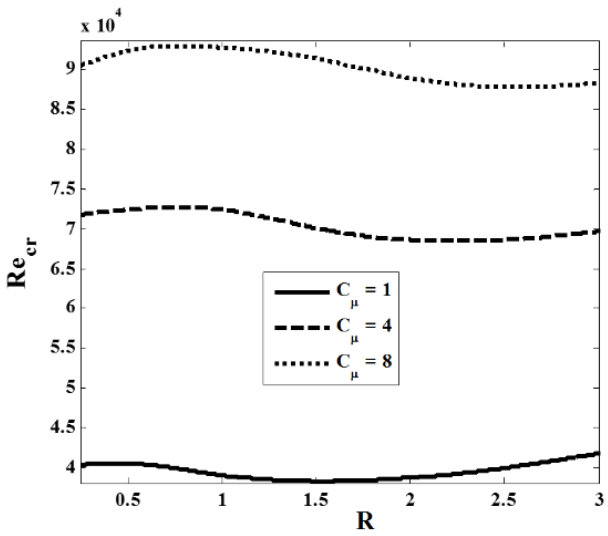

Fig. 12. Effect of the thickness ratio, $R$, on the critical Reynolds number $(\alpha=1.5, \xi=0.5)$.

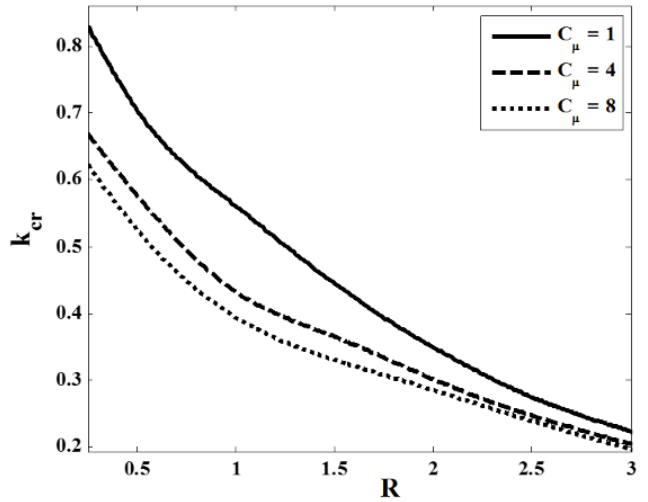

Fig. 13. Effect of the thickness ratio, $R$, on the critical wavenumber $(\alpha=1.5, \xi=0.5)$.

Figure 14 shows the effect of density ratio (defined as $\left.C_{r}=\rho_{2} / \rho_{1}\right)$ on the critical Reynolds number. An increase in the density of the Newtonian fluids has a destabilizing effect on the interface although its effect appears to asymptote to a limiting value. The wavenumbers are increased when the density of wall layer is increased. Since density plays no role on the fully-developed base-flow velocity profiles, its effect should be attributed to the inertial effects. 


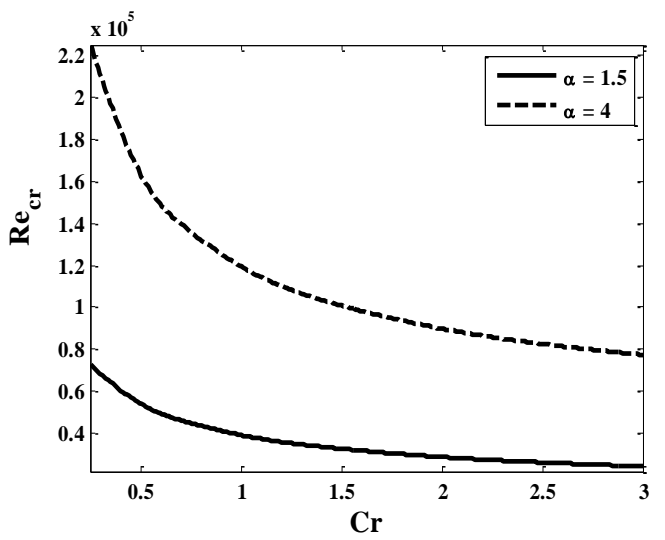

Fig. 14. Effect of the density ratio on the critical Reynolds number $\left(\mathrm{R}=1, \zeta=0.5, \mathrm{C}_{\mu}=1\right)$.

It needs to be mentioned that in real world, the density ratio of oil-to-water is often realized to be very close to one (usually less than one but for some extra-heavy oils it can be slightly above one). So, the results shown in this figure are of little consequence for true core-annular flows when transporting real waxy crude oils although such results can be very useful for cementing operation in drilling operation of oil wells.

\section{CONCLUding REMARKS}

In this study, the interfacial stability of a thixotropic fluid squeezed between two Newtonian fluids of the same viscosity and the same thickness flowing in a triple-layered configuration has been numerically studied using normal mode, temporal stability analysis. Assuming that the core fluid obeys Moore model, the effects of different parameters in this fluid model (notably, the thixotropy number and its viscosity-difference ratio) have been investigated on the unstable modes having assumed that the eigenmodes are symmetric. The reason for focusing on symmetric modes is the notion that they have been realized to be more dangerous than the other modes in core-annular flow (Joseph et al. 1984; Hu and Patankar, 1995). The symmetry of such eigenmodes allowed us to consider only half of the channel as the computational domain (i.e., the lower half) which significantly lowered the run time.

Based on the results obtained in this work, an increase in the thixotropy number (which is defined as the ratio of the fluid's time constant to the flow's time constant) lowers the critical Reynolds number. But, based on the definition of this dimensionless number, it can be concluded that the time constant introduced through thixotropic behavior of the Moore fluid has a stabilizing effect on the interface whereas the shear-thinning behavior of the Moore fluid has a destabilizing effect on the interface. An increase in the viscosity-difference ratio (which is attained by lowering the zero-shear viscosity of the Moore fluid or by increasing the viscosity of the wall layer) can stabilize or destabilize the interface depending on this ratio being smaller or larger than a threshold.
We also would like to stress that although the results obtained in the present work can be used for designing operations leading to the production of multi-layered sheets, it should be conceded that the results can be extended to the cementing operation of boreholes in drilling industry (i.e., where the working fluids are slightly miscible) and also to the pipeline transport of waxy crude oils in core-annular flow provided a hydrocarbon such as a kerosene is used as the lubricant. Having said this, it must be stressed that, the present study is more of a fundamental nature rather than industrial. That is to say that, no specific sheet material, cement slurry, drilling mud, or waxy oil has been used or considered in this study. In fact, such complex fluids usually obey rheological models which are much more complicated than the Moore model. In spite of its apparent simplicity, however, this simplistic fluid model has enabled us to differentiate thixotropic effects from shear-thinning effects and that is all we were looking for in the present work. In fact, the prediction that the sole qualitative effect of thixotropy on the interfacial instability is a stabilizing effect is the main contribution of the present work. In real world, however, the stabilizing effect of thixotropy should simultaneously compete with the destabilizing effect of shear-thinning and also any stabilizing/destabilizing effect which might be introduced through the working fluids' viscoelastic and/or viscoplastic effects. Such a thorough analysis requires more sophisticated rheological fluid models such as Dullaert-Mewis (Ahmadpour and Sadeghy, 2014) which should be done in future works.

Another direction for extending the present work is to consider non-symmetric modes. Obviously, a random disturbance can be decomposed into both symmetric and non-symmetric (say, even and odd) Fourier modes. And, as is well established in the literature, a base flow can be vulnerable to both types of modes. The fact is that, whenever a coreannular flow becomes unstable the instability exhibits itself first in an axisymmetric fashion (Preziosi et al. 1990). This is the main reason why we have focused on symmetric modes in our instability analysis. But, we are aware that under certain flow conditions non-axisymmtric modes might also become unstable in such flows. Obviously, the prediction that a fluid's thixotropic behavior can stabilize symmetric modes may not necessarily mean that thixotropy can also stabilize non-symmetric modes. So, future works should be directed towards addressing the stability of nonsymmetric modes on interfacial flows of thixotropic fluids, in general, and core-annular flows, in particular. The present work can be regarded as a good first step in that direction.

\section{ACKNOWLEDGEMENT}

The authors would like to thank the respectful reviewers for their constructive comments. This paper was written while K. Sadeghy was on study leave at the Department of Mechanical Engineering, University of British Columbia, Canada, and he 
would like to thank Prof. I. Frigaard for his invitation and hospitality. The financial support received by K. Sadeghy from the University of Tehran during his one-year sabbatical leave is also much appreciated.

\section{REFERENCES}

Ahmadpour, A. and K. Sadeghy (2014). Start-up flows of Dullaert-Mewis viscoplasticthixoelastic fluids: A two-dimensional analysis, J. Non-Newtonian Fluid Mech., 214, 1-17.

Anturkar, N. R, T. C. Papanastasiou and J. O. Wilkes (1990a). Linear stability analysis of multilayer plane Poiseuille flow, Physics of Fluids A, 2, 530-541.

Anturkar, N. R, T. C. Papanastasiou, J. O. Wilkes (1990b). Stability of multilayer extrusion of viscoelastic liquids, AIChE J 36, 710-724.

Barnes, H. A. (1997). Thixotropy - a review, Journal of Non-Newtonian Fluid Mechanics 70, 1-33.

Bellman, R. and R. H. Pennington (1954). Effects of surface tension and viscosity on Taylor instability, Quarterly of Applied Mathematics 12 (2), 151-162.

Billingham, J. and J. W. J. Ferguson (1993). Laminar, unidirectional flow of a thixotropic fluid in a circular pipe, Journal of NonNewtonian Fluid Mechanics 47, 21-55.

Chen, K. (1991). Interfacial instability due to elastic stratification in concentric coextrusion of two viscoelastic fluids. Journal of Non-Newtonian Fluid Mechanics 40, 155-175.

Chen, K. P. and Y. Zhang (1993). Stability of the interface in co-extrusion flow of two viscoelastic fluids through a pipe, Journal of Fluid Mechanics 247, 489-502.

Chen, K. and D. D. Joseph (1991). Long wave and lubrication theories for core-annular flow. Physics of Fluids A, 3, 2672-2679.

Cheng, D. C. H and F. Evans (1965). Phenomenological characterization of the rheological behaviour of inelastic reversible thixotropic and antithixotropic fluids, British Journal of Applied Physics 16.

Clark, A. F. and A. Shapiro (1949). Method of pumping viscous petroleum. U.S. Patent No. $2,533,878$.

Clement, C. (1979). A scientific approach to the use of thixotropic cement, SPE-6011-PA, Journal of Petroleum Technology 31 (3).

Drazin, P. G. and W. H. Reid (1981). Hydrodynamic Stability, Cambridge University Press, Cambridge, UK.

Foroushan, H. K., E. M. Ozbayoglu, S. Z. Miska, M. Yu and P. J. Gomes (2018). On the instability of the cement/fluid interface and fluid mixing, Society of Petroleum Engineers: SPE 180322PA, 33(1).
Frigaard, I. A. and J. P. Crawshaw (1999). Preventing buoyancy-driven flows of two Bingham fluids in a closed pipe - Fluid rheology design for oilfield plug cementing, Journal of Engineering Mathematics 36: 327348.

Ghosh, S., T. K. Mandal, G. Das, and P. K. Das (2009). Review of oil water core annular flow, Renewable and Sustainable Energy Reviews 13, 1957-1965.

Hickox, C. E. (1971). Instability due to viscosity and density stratification in axisymmetric pipe flow, Physics of Fluids 14(2), 251-262.

Hinch, E. J., O. J. Harris, and J. M. Rallison (1992). The instability mechanism for two elastic liquids being co-extruded. J. NonNewtonian Fluid Mech., 43, 311- 324.

Hooper, A.P., and W.G.C. Boyd (1983). Shear-flow instability at the interface between two viscous fluids, J. Fluid Mech, 128, 507-528.

Hormozi, S., D.M. Martinez, and I. Frigaard (2011). Stable core-annular flows of viscoelastic fluids using the visco-plastic lubrication technique. Journal of Non-Newtonian Fluid Mechanics $166,1356-1368$

$\mathrm{Hu}, \mathrm{H} . \mathrm{H}$. and N. Patankar (1995). Nonaxisymmetric instability of core-annular flow. Journal of Fluid Mechanics 290, 213-224.

Hu, H. H. and D. D. Joseph (1989). Lubricated pipkelining: stability of core-annular flow. Part 2. Journal of Fluid Mechanics 205, 359- 396.

Joseph, D. D., R. R. Bai, K. R. Chen, and Y. Y. Renardy (1997). Core-annular flows. Annual Review of Fluid Mechanics 29, 65-90.

Joseph, D. D., Y. Renardy and M. Renardy (1984). Instability of the flow of immiscible liquids with different viscosities in a pipe. Journal of Fluid Mechanics 141, 309-317.

Kan, M., M. Djabourov and J. L. Volle (2004). Rheology and structure of waxy crude oils in quiescent and under shearing conditions, Fuel $83,1591-1605$.

Livescu, S. (2012). Mathematical modeling of thixotropic drilling mud and crude oil flow in wells and pipelines-A review, Journal of Petroleum Science and Engineering 98-99: 174-184

Martinez-Palou, R., M. L. Mosqueira, B. ZapataRendon, E. Mar-Juarez, C. Bernal-Huicochea, J. C. Clavel-Lopez and J. Aburto (2011). Transportation of heavy and extra heavy crude oil by pipeline: a review, Journal of Petroleum Science and Engineering 75, 274-282.

Mewis, J. (1979). Thixotropy - a general review, Journal of Non-Newtonian Fluid Mechanics 6 , $1-20$.

Moore, F. (1959). The rheology of ceramic slips and bodies, Transactions and journal of the British 
M. Safarifard et al. / JAFM, Vol. 13, No. 5, pp. 1363-1375, 2020.

Ceramic Society 58, 470-494.

Preziosi, L., K. Chen and D. D. Joseph (1990). Lubricating pipelining: Stability of core-annular flow, Journal of Fluid Mechanics 201, 323-356.

Renardy, Y. (1985). Instability at the interface between two shearing fluids in a channel, Physics of Fluids 28, 3441.

Renardy, Y. (1988). Stability of the interface in twolayer Couette flow of upper convected Maxwell liquids. Physics of Fluids 14, 251-262.

Saffman, P. G., and G. Taylor (1958). The penetration of a fluid into a medium or HeleShaw cell containing a more viscous liquid, Proceedings of the Royal Society of London, Series A. 245, 312-329.

Sahu, K. C. (2019) Linear instability in a miscible core-annular flow of a Newtonian and a Bingham fluid, Journal of Non-Newtonian Fluid Mechanics 264, 159- 169.

Sarmadi, P. and I. A. Frigaard (2019). Stable coreannular horizontal flows in inaccessible domains via a triple-layer configuration, Chemical Engineering Science: X, 3, 100028: $1-12$.

Schrenk, W. J., N. L. Bradley, T. Alfrey Jr. and H.
Maack (1978). Interfacial flow instability in multilayer coextrusion, Polymer Engineering and Science 18(8), 620-623.

Su, Y. Y and B. Khomami (1991). Stability of multilayer power-law and second-order fluids in plane Poiseuille flow, Chemical Engineering Communications 109, 209-223.

Taghiloo, B., P. Sadeghi, P. Sarmadi, M. Saffaripour and K. Sadeghy (2019). Buoyancy-driven exchange flow of immiscible yield-stress fluids in a vertical closed-ended container, Journal of Non-Newtonian Fluid Mechanics 265, 79-91

Wardhaugh, L. T. and D. V. Boger (1991). Flow characteristics of waxy crude oils: application to pipeline design, AIChE Journal 37, 871-885.

Wei, H. H. (2005). Marangoni destabilization on a core-annular film flow due to the presence of surfactant, Physics of Fluids 17, 027101-9.

Yih, C. S. (1967). Instability due to viscosity stratification, Journal of Fluid Mechanics 27(2), 337-352.

Yu, H. S and E. M. Sparrow (1969). Experiments on two-component stratified flow in a horizontal duct, Trans. ASME. Journal of Heat Transfer 91, 51-58. 